\section{Tactile Agnosia}

Kerry Donnelly

VA WNY Healthcare System, University of Buffalo (SUNY) Behavioral Health Careline (116B), Buffalo, NY, USA

\section{Synonyms}

Haptic agnosia; Tactile asymbolia; Tactile object agnosia; Tactoagnosia

\section{Definition}

Tactile agnosia is the inability to recognize or identify an object by naming, matching to sample, or demonstrating its use by solely tactual manipulations, despite intact elementary tactile perceptions. Thus, by definition, the patient may be able to describe certain physical characteristics of the object by touch alone, such as size, shape, and texture, but not identify it. Tactile agnosia in its pure form (asymbolia) is considered rare and is somewhat controversial, but if present, is thought to be associated with lesions in the posterior parietal lobe in either hemisphere. The deficit may be unilateral or bilateral. If an object cannot be named when placed in the left hand, but recognition is possible through demonstration of use or matching to sample, then a callosal disconnection to the language areas of the left hemisphere needs to be considered. If a similar limitation is found in both hands, an aphasic deficit has to be ruled out. In some cases, the object may not be immediately recognized but its identity may be slowly deduced from an analysis of its elementary characteristics.

\section{Cross-References}

- Agnosia

- Astereognosis

- Tactile Asymboly

\section{References and Readings}

Platz, T. (1996). Tactile agnosia: Casuistic evidence and theoretical remarks on modality-specific meaning representations and sensorimotor integration. Brain, 119, 1565-1574.

Reed, C. L., Caselli, R. J., \& Farah, M. J. (1996). Tactile agnosia: Underlying impairment and implications for normal tactile object recognition. Brain, 119, 875-888. 